\title{
Discrete Approximations of Determinantal Point Processes on Continuous Spaces: Tree Representations and Tail Triviality
}

\author{
Hirofumi Osada ${ }^{1}$ (D) . Shota Osada ${ }^{1}$
}

Received: 21 August 2017 / Accepted: 21 November 2017 / Published online: 28 November 2017

(C) The Author(s) 2017. This article is an open access publication

\begin{abstract}
We prove tail triviality of determinantal point processes $\mu$ on continuous spaces. Tail triviality has been proved for such processes only on discrete spaces, and hence we have generalized the result to continuous spaces. To do this, we construct tree representations, that is, discrete approximations of determinantal point processes enjoying a determinantal structure. There are many interesting examples of determinantal point processes on continuous spaces such as zero points of the hyperbolic Gaussian analytic function with Bergman kernel, and the thermodynamic limit of eigenvalues of Gaussian random matrices for $\mathrm{Sine}_{2}, \mathrm{Airy}_{2}, \mathrm{Bessel}_{2}$, and Ginibre point processes. Our main theorem proves all these point processes are tail trivial.
\end{abstract}

Keywords Determinantal point processes - Tail triviality $\cdot$ Tree representations $\cdot$ Random matrices

Mathematics Subject Classification 60B20 $\cdot 82 \mathrm{C} 22 \cdot 82 \mathrm{~B} 21 \cdot 60 \mathrm{~K} 35$

\section{Introduction}

Let $S$ be a locally compact, complete, separable metric space with metric $\mathrm{d}(\cdot, \cdot)$. We assume $S$ is unbounded. We equip $S$ with a Radon measure $\mathrm{m}$ such that $\mathrm{m}(\mathcal{O})>0$ for any non-empty open set $\mathcal{O}$ in $S$. Let $S$ be the configuration space over $S$ (see (2.1) for definition). $S$ is a Polish space equipped with the vague topology.

$凶$ Hirofumi Osada

osada@math.kyushu-u.ac.jp

Shota Osada

osada@kyudai.jp

1 Faculty of Mathematics, Kyushu University, Fukuoka 819-0395, Japan 
A determinantal point process $\mu$ on $S$ is a probability measure on $(\mathrm{S}, \mathcal{B}(\mathrm{S})$ ) for which the $m$-point correlation function $\rho^{m}$ with respect to $\mathrm{m}$ is given by the determinant

$$
\rho^{m}(\mathbf{x})=\operatorname{det}\left[\mathrm{K}\left(x_{i}, x_{j}\right)\right]_{i, j=1}^{m} .
$$

Here $\mathrm{K}: S \times S \rightarrow \mathbb{C}$ is a measurable kernel and $\mathbf{x}=\left(x_{1}, \ldots, x_{m}\right)$. We refer to Sects. 2 and e.g. $[1,3,10]$ for the definition of correlation functions and related notions. $\mu$ is said to be associated with $(\mathrm{K}, \mathrm{m})$ and also a $(\mathrm{K}, \mathrm{m})$-determinantal point process.

We set $\mathrm{K} f(x)=\int_{S} \mathrm{~K}(x, y) f(y) \mathrm{m}(d y)$. We regard $\mathrm{K}$ as an operator on $L^{2}(S, \mathrm{~m})$ and denote it by the same symbol. We say $\mathrm{K}$ is of locally trace class if $\mathrm{K}_{A} f(x)=$ $\int 1_{A}(x) \mathrm{K}(x, y) 1_{A}(y) f(y) \mathrm{m}(d y)$ is a trace class operator on $L^{2}(S, \mathrm{~m})$ for any compact set $A$.

Throughout this paper, we assume that $\mathrm{K}$ satisfies:

(A1) $\mathrm{K}$ is bounded, Hermitian symmetric, of locally trace class, and $\operatorname{Spec}(\mathrm{K}) \subset[0,1]$.

From (A1) we deduce that the associated determinantal point process $\mu=\mu^{\mathrm{K}, \mathrm{m}}$ exists and is unique $[7,10,12]$.

In the last two decades, determinantal point processes have been extensively studied. They contain many interesting examples; e.g., spanning trees and Schur measures on discrete spaces, zero points of the hyperbolic Gaussian analytic function with Bergman kernel, and thermodynamic limits of eigenvalues of Gaussian random matrices such as Sine $_{2}$, Airy $_{2}$, Bessel $_{2}$, and Ginibre point processes on continuous spaces $[1,5,10]$.

Determinantal point processes on discrete spaces have a well-behaved algebraic structure; as a result, some important facts are only known for discrete determinantal point processes $[4,6-8,12]$. One such example is tail triviality, which says that each event of a tail $\sigma$-field Tail(S) takes value 0 or 1 . We refer to (2.3) for the definition of Tail(S).

The purpose of this paper is to prove that the tail $\sigma$-field Tail(S) of $S$ is trivial with respect to $\mu$. If the space $S$ is discrete, then tail triviality has been proved by Shirai-Takahashi [11] for $\operatorname{Spec}(\mathrm{K}) \subset(0,1)$, and by Russell Lyons [7] for $\operatorname{Spec}(\mathrm{K}) \subset[0,1]$. If the space $S$ is continuous, the problem remained open [8].

To prove tail triviality we introduce a discrete approximation for determinantal point processes, called the tree representation. This representation has a determinantal structure, and so belongs to determinantal point processes on discrete spaces.

A m-partition $\Delta=\left\{\mathcal{A}_{i}\right\}_{i \in I}$ of $S$ is a countable collection of disjoint relatively compact, measurable subsets of $S$ such that $\cup_{i} \mathcal{A}_{i}=S$ and that $\mathrm{m}\left(\mathcal{A}_{i}\right)>0$ for all $i \in I$. For two partitions $\Delta=\left\{\mathcal{A}_{i}\right\}_{i \in I}$ and $\Gamma=\left\{\mathcal{B}_{j}\right\}_{j \in J}$, we write $\Delta \prec \Gamma$ if for each $j \in J$ there exists $i \in I$ such that $\mathcal{B}_{j} \subset \mathcal{A}_{i}$. We assume:

(A2) There exists a sequence of m-partitions $\{\Delta(\ell)\}_{\ell \in \mathbb{N}}$ satisfying (1.2)-(1.4).

$$
\begin{aligned}
& \Delta(\ell) \prec \Delta(\ell+1) \quad \text { for all } \ell \in \mathbb{N}, \\
& \sigma\left[\bigcup_{\ell \in \mathbb{N}} \mathcal{F}_{\ell}\right]=\mathcal{B}(S), \\
& \#\left\{j ; \mathcal{A}_{\ell+1, j} \subset \mathcal{A}_{\ell, i}\right\}=2 \text { for all } i \in I(\ell) \text { and } \ell \in \mathbb{N},
\end{aligned}
$$

where we set $\Delta(\ell)=\left\{\mathcal{A}_{\ell, i}\right\}_{i \in I(\ell)}$ and $\mathcal{F}_{\ell}:=\mathcal{F}_{\Delta(\ell)}=\sigma\left[\mathcal{A}_{\ell, i} ; i \in I(\ell)\right]$. Furthermore, \#\{.\} denotes the cardinality of $\{\cdot\}$.

Condition (1.4) is just for simplicity. This condition implies that the sequence $\{\Delta(\ell)\}_{\ell \in \mathbb{N}}$ has a binary tree-like structure. We remark that (A2) is a mild assumption and, indeed, satisfied if $S$ is an open set in $\mathbb{R}^{d}$ and $\mathrm{m}$ has positive density with respect to the Lebesgue measure. We now state one of our main theorems: 
Theorem 1 Assume (A1) and (A2). Let $\mu$ be the (K, $\mathrm{m})$-determinantal point process. Then $\mu$ has a trivial tail. That is, $\mu(A) \in\{0,1\}$ for all $A \in$ Tail(S).

Many interesting determinantal point processes arise from random matrices such as Sine $_{2}$, Airy 2 , and Bessel 2 point processes in $\mathbb{R}$ and the Ginibre point process in $\mathbb{R}^{2}$. Applying Theorem 1 to these examples we obtain that all have trivial tails. We shall present these examples in Sect. 6.

We now explain the idea of the proof. We have two candidates for the discrete approximations of $\mu$. One is the approximation of the kernel K. Let $\mathrm{K}_{\ell}(x, y)$ be the discrete kernel on $I(\ell)$ such that

$$
\mathrm{K}_{\ell}(x, y)=\frac{1}{\mathrm{~m}\left(\mathcal{A}_{\ell}(x)\right) \mathrm{m}\left(\mathcal{A}_{\ell}(y)\right)} \int_{\mathcal{A}_{\ell}(x) \times \mathcal{A}_{\ell}(y)} \mathrm{K}(u, v) \mathrm{m}(d u) \mathrm{m}(d v),
$$

where $\mathcal{A}_{\ell}(x)$ is such that $x \in \mathcal{A}_{\ell}(x) \in \Delta(\ell)$. Then $\mathrm{K}_{\ell}$ can be regarded as a discrete kernel on $I(\ell)$. If $\mathrm{K}_{\ell}$ satisfies (A1), then $\mathrm{K}_{\ell}$ generates determinantal point field $\mu_{\mathrm{K}_{\ell}}$. Indeed, $\operatorname{Spec}\left(\mathrm{K}_{\ell}\right) \subset$ $[0,1]$ follows from $\operatorname{Spec}(K) \subset[0,1]$ and the Fubini theorem. One can expect the convergence of the kernel $\mathrm{K}_{\ell}$ to $\mathrm{K}$, and as a result, the weak convergence of $\mu_{\mathrm{K}_{\ell}}$ to $\mu$, at least for continuous $\mathrm{K}$. Because $\mu_{\mathrm{K}_{\ell}}$ is a determinantal point process on the discrete space, its tail $\sigma$-field is trivial. Such weak convergence, however, does not suffice for the convergence of the values on the tail $\sigma$-field Tail(S).

Taking the above into account, we consider the second approximation given by $\mu\left(\cdot \mid \mathcal{G}_{\ell}\right)$ below. Let $\mathcal{G}_{\ell}$ be the sub- $\sigma$-field of $\mathcal{B}(\mathrm{S})$ given by

$$
\mathcal{G}_{\ell}=\sigma\left[\left\{\mathrm{S} \in \mathrm{S} ; \mathrm{S}\left(\mathcal{A}_{\ell, i}\right)=n\right\} ; i \in I(\ell), n \in \mathbb{N}\right] .
$$

Combining (1.2) and (1.3) with (1.5), we obtain

$$
\mathcal{G}_{\ell} \subset \mathcal{G}_{\ell+1}, \quad \sigma\left[\mathcal{G}_{\ell} ; \ell \in \mathbb{N}\right]=\mathcal{B}(\mathrm{S}) .
$$

Let $\mu\left(\cdot \mid \mathcal{G}_{\ell}\right)$ be the regular conditional probability of $\mu$ with respect to $\mathcal{G}_{\ell}$. Using (1.6), we shall prove in Lemma 6 that for all $U \in \mathcal{B}(\mathrm{S})$

$$
\lim _{\ell \rightarrow \infty} \mu\left(U \mid \mathcal{G}_{\ell}\right)(\mathbf{s})=1_{U}(\mathbf{s}) \quad \text { for } \mu \text {-a.s. s. }
$$

We see that the convergence in (1.7) is stronger than the weak convergence. In particular, the convergence in (1.7) is valid for all $U \in$ Tail(S) because Tail(S) $\subset \mathcal{B}(\mathrm{S})$.

We can naturally regard $\Delta(\ell)=\left\{\mathcal{A}_{\ell, i}\right\}_{i \in I(\ell)}$ as a discrete, countable set with the interpretation that each element $\mathcal{A}_{\ell, i}$ is a point. Thus, $\mu\left(\cdot \mid \mathcal{G}_{\ell}\right)$ can be regarded as a point process on the discrete set $\Delta(\ell)$.

If $\mu\left(\cdot \mid \mathcal{G}_{\ell}\right)$ were a determinantal point process for each $\ell$, then Theorem 1 would follow from (1.7) immediately because determinantal point processes on discrete spaces always have trivial tails, and as discussed above, $\mu\left(\cdot \mid \mathcal{G}_{\ell}\right)$ is naturally regarded as a determinantal point process on the discrete space $\Delta(\ell)$. This is clearly not the case because determinantal point processes are supported on single configurations and

$$
\mu\left(\left\{\mathrm{s} ; \mathrm{s}\left(\mathcal{A}_{\ell, i}\right) \geq 2\right\} \mid \mathcal{G}_{\ell}\right)>0 .
$$

Hence we introduce a sequence of fiber bundle-like sets $\mathbb{I}(\ell)(\ell \in \mathbb{N})$ in Sect. 2 with base space $\Delta(\ell)$ with fiber consisting of a set of binary trees. We further expand $\mathbb{I}(\ell)$ to $\Omega(\ell)$ in (2.27), which has a fiber whose element is a product of a tree $i$ and a component $\mathcal{B}_{\ell, i}$ of partitions. See notation after Theorem 2.

Let $\left.\mu\right|_{\mathcal{G}_{\ell}}$ denote the restriction of $\mu$ on $\mathcal{G}_{\ell}$. By construction $\left.\mu\right|_{\mathcal{G}_{\ell}}(\mathrm{A})=\mu\left(\mathrm{A} \mid \mathcal{G}_{\ell}\right)$ for all $\mathrm{A} \in \mathcal{G}_{\ell}$. In Theorems 2 and 3 , we construct a lift $\nu_{\mathbb{F}(\ell)} \diamond \mathrm{m}_{\mathbb{F}(\ell)}$ of $\left.\mu\right|_{\mathcal{G}_{\ell}}$ on the fiber bundle 
$\Omega(\ell)$, and prove tail triviality of the lift $v_{\mathbb{F}(\ell)} \diamond \mathrm{m}_{\mathbb{F}(\ell)}$ in Theorem 5, which establishes tail triviality of $\left.\mu\right|_{\mathcal{G}_{\ell}}$ in Theorem 6 . Combining Theorem 6 with the martingale convergence theorem in Lemma 6, we obtain Theorem 1.

The key point of the construction of the lift $\nu_{\mathbb{F}(\ell)} \diamond \mathrm{m}_{\mathbb{F}(\ell)}$ is that we construct a consistent family of orthonormal bases $\mathbb{F}(\ell)=\left\{f_{\ell, i}\right\}_{i \in \mathbb{I}(\ell)}$ in (2.15) and (2.16), and that we introduce the kernel $\mathrm{K}_{\mathbb{F}(\ell)}$ on $\mathbb{I}(\ell)$ in (2.21) such that

$$
\mathrm{K}_{\mathbb{F}(\ell)}(i, j)=\int_{S \times S} \mathrm{~K}(x, y) \overline{f_{\ell, i}(x)} f_{\ell, j}(y) \mathrm{m}(d x) \mathrm{m}(d y) .
$$

We shall prove in Lemma 2 that $\mathrm{K}_{\mathbb{F}(\ell)}$ is a determinantal kernel on $\mathbb{I}(\ell)$, and present $v_{\mathbb{F}(\ell)}$ as the associated determinantal point process on $\mathbb{I}(\ell)$. To some extent, $v_{\mathbb{F}(\ell)}$ is isometric to $\left.\mu\right|_{\mathcal{G}_{\ell}}$ through the orthonormal basis $\mathbb{F}(\ell)=\left\{f_{\ell, i}\right\}_{i \in \mathbb{I}(\ell)}$. We shall indeed prove in Theorem 2 that their correlation functions $\rho_{\mathcal{G}_{\ell}}^{m}$ and $\rho_{\mathbb{F}(\ell)}^{m}$ satisfy the identity:

$$
\int_{\mathbb{A}} \rho_{\mathcal{G}_{\ell}}^{m}(\mathbf{x}) \mathrm{m}^{m}(d \mathbf{x})=\sum_{\mathbf{i} \in \mathbb{I}_{\ell}(\mathbb{A})} \rho_{\mathbb{F}(\ell)}^{m}(\mathbf{i}),
$$

which is a key to construct the lift $\nu_{\mathbb{F}(\ell)} \diamond \mathrm{m}_{\mathbb{F}(\ell)}$.

While preparing the manuscript, we have heard that Professor A. Bufetov has proved independently tail triviality of determinantal point processes on continuous spaces independently of us (a seminar talk at Kyushu University in October 2015). His method is completely different from ours and requires a restriction on an integrability condition of the determinantal kernel $\mathrm{K}(x, y)$. An improved version of the work is now available in [2].

The organization of the paper is as follows. In Sect. 2, we introduce definitions and concepts and state the main theorems (Theorems 2-6). We give tree representations of $\mu$. In Sect. 3, we prove Theorem 2. In Sect. 4, we prove Theorems 3-6. In Sect. 5, we prove Theorem 1. In Sect. 6, we present motivational examples such as $\mathrm{Sine}_{2}, \mathrm{Airy}_{2}$, and Bessel 2 , and Ginibre point processes.

\section{Set Up and Main Results}

In this section, we recall various essentials and present the main theorems (Theorems 2-6) other than Theorem 1.

A configuration space $S$ over $S$ is a set consisting of configurations on $S$ such that

$$
\mathrm{S}=\left\{\mathrm{s} ; \mathrm{s}=\sum_{i} \delta_{s_{i}},\left\{s_{i}\right\} \subset S, \mathrm{~s}(K)<\infty \text { for any compact } K\right\},
$$

where $\delta_{s_{i}}$ denotes the delta measure at $s_{i}$. A probability measure $\mu$ on $(\mathrm{S}, \mathcal{B}(\mathrm{S}))$ is called a point process, also called random point field. A symmetric function $\rho^{m}$ on $S^{m}$ is called the $m$-point correlation function of a point process $\mu$ with respect to a Radon measure $\mathrm{m}$ if it satisfies

$$
\int_{\mathbf{S}} \prod_{i=1}^{j} \frac{\mathbf{s}\left(A_{i}\right) !}{\left(\mathbf{s}\left(A_{i}\right)-k_{i}\right) !} \mu(d \mathbf{s})=\int_{A_{1}^{k_{1}} \times \cdots \times A_{j}^{k_{j}}} \rho^{m}(\mathbf{x}) \mathbf{m}^{m}(d \mathbf{x}) .
$$

Here $A_{1}, \ldots, A_{j} \in \mathcal{B}(S)$ are disjoint and $k_{1}, \ldots, k_{j} \in \mathbb{N}$ such that $k_{1}+\cdots+k_{j}=m$. If $\mathrm{s}\left(A_{i}\right)-k_{i} \leq 0$, we set $\mathbf{s}\left(A_{i}\right) ! /\left(\mathbf{s}\left(A_{i}\right)-k_{i}\right) !=0$. 
We fix a point $o \in S$ as the origin, and set $S_{r}=\{x \in S ; \mathrm{d}(o, x)<r\}$. Each $S_{r}$ is assumed to be relatively compact, and thus $\mathrm{S}\left(S_{r}\right)<\infty$ for all $\mathrm{S} \in \mathrm{S}$ and $r \in \mathbb{N}$. In this sense, each element $S$ of $S$ is a locally finite configuration. We note that this notion depends on the choice of metric d on $S$.

For a Borel set $A$ we set $\pi_{A}: \mathrm{S} \rightarrow \mathrm{S}$ by $\pi_{A}(\mathrm{~s})(\cdot)=\mathrm{s}(\cdot \cap A)$. We set $\pi_{S_{r}^{c}}: \mathrm{S} \rightarrow \mathrm{S}$ such that $\pi_{S_{r}^{c}}(\mathrm{~S})=\mathrm{S}\left(\cdot \cap S_{r}^{c}\right)$. We denote by Tail(S) the tail $\sigma$-field such that

$$
\operatorname{Tail}(\mathrm{S})=\bigcap_{\mathrm{r}=1}^{\infty} \sigma\left[\pi_{S_{r}^{c}}\right] .
$$

If we replace $S_{r}$ by any increasing sequence $\left\{O_{\mathrm{r}}\right\}$ of relatively compact open sets such that $\cup_{\mathrm{r}=1}^{\infty} O_{\mathrm{r}}=S$, then Tail(S) defines the same $\sigma$-field. Thus Tail(S) is independent of the choice of $\left\{O_{\mathrm{r}}\right\}$.

Let $\Delta(\ell)=\left\{\mathcal{A}_{\ell, i}\right\}_{i \in I(\ell)}$ be as in (A2), where $\ell \in \mathbb{N}$. We set $\Delta=\left\{\mathcal{A}_{i}\right\}_{i \in I}$ such that

$$
\Delta=\Delta(1), \quad \mathcal{A}_{i}=\mathcal{A}_{1, i} \quad I=I(1) .
$$

In consequence of (1.4), we assume without loss of generality that each element $i$ of the parameter set $I(\ell)$ is of the form

$$
I(\ell)=I \times\{0,1\}^{\ell-1} .
$$

That is, each $i \in I(\ell)$ is of the form $i=\left(j_{1}, \ldots, j_{\ell}\right) \in I \times\{0,1\}^{\ell-1}$. We take a label $i \in \cup_{\ell=1}^{\infty} I(\ell)$ in such a way that, for $\ell<\ell^{\prime}, i \in I(\ell)$, and $i^{\prime} \in I\left(\ell^{\prime}\right)$,

$$
\mathcal{A}_{\ell, i} \supset \mathcal{A}_{\ell^{\prime}, i^{\prime}} \Leftrightarrow i=\left(j_{1}, \ldots, j_{\ell}\right) \text { and } i^{\prime}=\left(j_{1}, \ldots, j_{\ell}, \ldots, j_{\ell^{\prime}}\right) .
$$

We denote by $\widetilde{\mathbb{I}}$ the set of all such parameters:

$$
\widetilde{\mathbb{I}}=\bigcup_{\ell=1}^{\infty} I(\ell)=\bigcup_{\ell=1}^{\infty} I \times\{0,1\}^{\ell-1} .
$$

We can regard $\widetilde{I}$ as a collection of binary trees and $I$ is the set of their roots.

Example 1 (Binary partitions of $\mathbb{R}$ ) Typically we can take $S=\mathbb{R}, \mathrm{m}(d x)=d x$, and $I=\mathbb{Z}$. For $i=\left(j_{1}, \ldots, j_{\ell}\right) \in I(\ell)$, we set $J_{1, i}=j_{1}$ and, for $\ell \geq 2$,

$$
J_{\ell, i}=j_{1}+\sum_{n=1}^{\ell-1} \frac{j_{n}}{2^{n}} .
$$

We take $\mathcal{A}_{\ell, i}=\left[J_{\ell, i}, J_{\ell, i}+2^{-\ell+1}\right)$.

For $i=\left(j_{1}, \ldots, j_{\ell}\right) \in \widetilde{\mathbb{I}}$, we set $\operatorname{rank}(i)=\ell$. For $i$ with $\operatorname{rank}(i)=\ell$, we set

$$
\mathcal{B}_{i}= \begin{cases}\mathcal{A}_{1, i} & \ell=1, \\ \mathcal{A}_{\ell-1, i^{-}} & \ell \geq 2,\end{cases}
$$

where $i^{-}=\left(j_{1}, \ldots, j_{\ell-1}\right)$ for $i=\left(j_{1}, \ldots, j_{\ell}\right) \in I(\ell)$. Let $\mathbb{I} \subset \widetilde{\mathbb{I}}$ such that

$$
\mathbb{I}=I \cup\left\{\bigcup_{\ell=2}^{\infty}\left\{i \in I(\ell) ; j_{\ell}=0\right\}\right\},
$$

where $i=\left(j_{1}, \ldots, j_{\ell}\right) \in I(\ell)$. 
Let $\mathbb{F}=\left\{f_{i}\right\}_{i \in \mathbb{I}}$ be an orthonormal basis of $L^{2}(S, \mathrm{~m})$ satisfying

$$
\begin{array}{ll}
\sigma\left[f_{i} ; i \in \mathbb{I}, \operatorname{rank}(i)=\ell\right]=\mathcal{F}_{\ell} & \text { for each } \ell \in \mathbb{N}, \\
\operatorname{supp}\left(f_{i}\right)=\mathcal{B}_{i} & \text { for each } i \in \mathbb{I}, \\
f_{i}(x)=1_{\mathcal{A}_{i}}(x) / \sqrt{\mathrm{m}\left(\mathcal{A}_{i}\right)} & \text { for } \operatorname{rank}(i)=1 .
\end{array}
$$

For a given sequence of m-partitions satisfying (A2), such an orthonormal basis exists. We present here an example.

Example 2 (Haar functions) We make the same assumptions as in Example 1. Let $i=$ $\left(j_{1}, \ldots, j_{\ell}\right) \in \mathbb{I}$. We set for, $\ell=1$ and $i=\left(j_{1}\right)$,

$$
f_{i}(x)=1_{\left[j_{1}, j_{1}+1\right)}(x)
$$

and, for $\ell \geq 2$ and $i=\left(j_{1}, \ldots, j_{\ell}\right) \in \mathbb{I}$,

$$
f_{i}(x)=2^{(\ell-1) / 2}\left\{1_{\left[J_{\ell, i}, J_{\ell, i}+2^{-\ell+1}\right)}(x)-1_{\left[J_{\ell, i}+2^{-\ell+1}, J_{\ell, i}+2^{-\ell+2}\right)}(x)\right\} .
$$

We can easily see that $\left\{f_{i}\right\}_{i \in \mathbb{I}}$ is an orthonormal basis of $L^{2}(\mathbb{R}, d x)$. We remark that $j_{\ell}=0$ because $i=\left(j_{1}, \ldots, j_{\ell}\right) \in \mathbb{I}$ as we set in (2.8).

We next introduce the $\ell$-shift of above objects such as $\mathbb{I}, \mathcal{B}_{i}$, and $\mathbb{F}=\left\{f_{i}\right\}_{i \in \mathbb{I}}$. Let $\widetilde{\mathbb{I}}(1)=\widetilde{\mathbb{I}}$ and, for $\ell \geq 2$,

$$
\widetilde{\mathbb{I}}(\ell):=\bigcup_{r=1}^{\infty} I(\ell) \times\{0,1\}^{r-1},
$$

where $I(\ell)=I \times\{0,1\}^{\ell-1}$ is as in (2.4). For $\ell, r \in \mathbb{N}$, we set $\theta_{\ell-1, r}: \widetilde{\mathbb{I}} \rightarrow \widetilde{\mathbb{I}}(\ell)$ such that $\theta_{0, r}=\mathrm{id}(\ell=1)$ and, for $\ell \geq 2$,

$$
\theta_{\ell-1, r}\left(\left(j_{1}, \ldots, j_{\ell+r-1}\right)\right)=\left(\mathbf{j}_{\ell}, j_{\ell+1}, \ldots, j_{\ell+r-1}\right) \in I(\ell) \times\{0,1\}^{r-1},
$$

where $\mathbf{j}_{\ell}=\left(j_{1}, \ldots, j_{\ell}\right) \in I(\ell)$. For $\ell=1$, we set $\mathbb{I}(1)=\mathbb{I}$. For $\ell \geq 2$, we set

$$
\mathbb{I}(\ell)=I(\ell) \cup\left\{\bigcup_{r=2}^{\infty} \theta_{\ell-1, r}(\mathbb{I})\right\} .
$$

We set $\operatorname{rank}(i)=r$ for $i \in I(\ell) \times\{0,1\}^{r-1}$. By construction $\operatorname{rank}(i)=r$ for $i \in \theta_{\ell-1, r}(\widetilde{\mathbb{I}})$. Let $\mathbb{F}(\ell)=\left\{f_{\ell, i}\right\}_{i \in \mathbb{I}(\ell)}$ such that, for $r=\operatorname{rank}(i)$,

$$
\begin{array}{ll}
f_{\ell, i}(x)=1_{\mathcal{A}_{\ell, i}(x) / \sqrt{\mathrm{m}\left(\mathcal{A}_{\ell, i}\right)}} & \text { for } r=1, \\
f_{\ell, i}(x)=f_{\theta_{\ell-1, r}^{-1}(i)}(x) & \text { for } r \geq 2,
\end{array}
$$

where $\Delta(\ell)=\left\{\mathcal{A}_{\ell, i}\right\}_{i \in I(\ell)}$ is given in (A2). Then $\mathbb{F}(\ell)=\left\{f_{\ell, i}\right\}_{i \in \mathbb{I}(\ell)}$ is an orthonormal basis of $L^{2}(S, \mathrm{~m})$. This follows from assumptions (2.15) and (2.16) and the fact that $\mathbb{F}=\left\{f_{i}\right\}_{i \in \mathbb{I}}$ is an orthonormal basis.

Remark 1 (1) We note that $f_{\ell, i} \in \mathbb{F}(\ell)$ is a newly defined function if $\operatorname{rank}(i)=1$, whereas $f_{\ell, i} \in \mathbb{F}(\ell)$ is an element of $\mathbb{F}$ if $\operatorname{rank}(i) \geq 2$. In particular, we see that

$$
\left\{f_{\ell, i}\right\}_{i \in \mathbb{I}(\ell), \operatorname{rank}(i) \geq 2} \subset\left\{f_{i}\right\}_{i \in \mathbb{I}, \operatorname{rank}(i) \geq 2} .
$$


(2) Let $j=\left(j_{1}, \ldots, j_{\ell+r-1}\right) \in \mathbb{I}$ and $i=\left(\mathbf{j}_{\ell}, j_{\ell+1}, \ldots, j_{\ell+r-1}\right) \in \mathbb{I}(\ell)$. Then

$$
j=\theta_{\ell-1, r}^{-1}(i) .
$$

Furthermore, $f_{\ell, i} \in \mathbb{F}(\ell)$ and $f_{j} \in \mathbb{F}$ satisfy $f_{\ell, i}=f_{j}$ for $r=\operatorname{rank}(i) \geq 2$.

(3) By construction, we see that

$$
\begin{array}{ll}
\sigma\left[f_{\ell, i} ; i \in \mathbb{I}(\ell), \operatorname{rank}(i)=r\right]=\mathcal{F}_{\ell-1+r} & \text { for each } \ell, r \in \mathbb{N}, \\
\operatorname{supp}\left(f_{\ell, i}\right)=\mathcal{B}_{\ell, i} & \text { for all } i \in \mathbb{I}(\ell),
\end{array}
$$

where we set, for $j=\theta_{\ell-1, r}^{-1}(i) \operatorname{such}$ that $\operatorname{rank}(i)=r$,

$$
\mathcal{B}_{\ell, i}=\mathcal{B}_{j}
$$

Using the orthonormal basis $\mathbb{F}(\ell)=\left\{f_{\ell, i}\right\}_{i \in \mathbb{I}(\ell)}$, we set $\mathrm{K}_{\mathbb{F}(\ell)}$ on $\mathbb{I}(\ell)$ by

$$
\mathrm{K}_{\mathbb{F}(\ell)}(i, j)=\int_{S \times S} \mathrm{~K}(x, y) \overline{f_{\ell, i}(x)} f_{\ell, j}(y) \mathrm{m}(d x) \mathrm{m}(d y) .
$$

Let $\lambda_{\mathbb{I}(\ell)}$ be the counting measure on $\mathbb{I}(\ell)$. We shall prove in Lemma 2 that $\left(\mathrm{K}_{\mathbb{F}(\ell)}, \lambda_{\mathbb{I}(\ell)}\right)$ satisfies (A1). Hence we obtain the associated determinantal point process $v_{\mathbb{F}(\ell)}$ on $\mathbb{I}(\ell)$ from general theory $[10,12]$.

For $i \in \mathbb{I}(\ell)$, let $\mathrm{m}_{f_{\ell, i}}(d x)$ be the probability measure on $S$ such that

$$
\mathrm{m}_{f_{\ell, i}}(d x)=\left|f_{\ell, i}(x)\right|^{2} \mathrm{~m}(d x) .
$$

For $\mathbf{i}=\left(i_{n}\right)_{n=1}^{m} \in \mathbb{I}(\ell)^{m}$ and $\mathbf{x}=\left(x_{n}\right)_{n=1}^{m}$, where $m \in \mathbb{N} \cup\{\infty\}$, we set

$$
\mathrm{m}_{f_{\ell, \mathbf{i}}}(d \mathbf{x})=\prod_{n=1}^{m}\left|f_{\ell, i_{n}}\left(x_{n}\right)\right|^{2} \mathrm{~m}\left(d x_{n}\right) .
$$

By (2.16) $\mathrm{m}_{f_{\ell, \mathrm{i}}}$ is a probability measure on $S^{m}$. By (2.19), we have

$$
\mathrm{m}_{f_{\ell, \mathbf{i}}}\left(\prod_{n=1}^{m} \mathcal{B}_{\ell, i_{n}}\right)=1 .
$$

Let $\mathcal{G}_{\ell}$ be the sub- $\sigma$-field as in (1.5). Let $v_{\mathbb{F}(\ell)}$ be the $\left(\mathrm{K}_{\mathbb{F}(\ell)}, \lambda_{\mathbb{I}(\ell)}\right)$-determinantal point process as before. Let $\rho_{\mathcal{G}_{\ell}}^{m}$ and $\rho_{\mathbb{F}(\ell)}^{m}$ be the $m$-point correlation functions of $\left.\mu\right|_{\mathcal{G}_{\ell}}$ and $\nu_{\mathbb{F}(\ell)}$ with respect to $m$ and $\lambda_{\mathbb{I}(\ell)}$, respectively. We now state one of our main theorems:

Theorem 2 Let $\mathbb{I}_{\ell}(\mathcal{A})=\left\{i \in \mathbb{I}(\ell) ; \mathcal{B}_{\ell, i} \subset \mathcal{A}\right\}$. For $\mathbb{A}=\mathcal{A}_{1} \times \cdots \times \mathcal{A}_{m}$, we set

$$
\mathbb{I}_{\ell}(\mathbb{A})=\mathbb{I}_{\ell}\left(\mathcal{A}_{1}\right) \times \cdots \times \mathbb{I}_{\ell}\left(\mathcal{A}_{m}\right) .
$$

Assume that $\mathcal{A}_{n} \in \Delta(\ell)$ for all $n=1, \ldots, m$. Then

$$
\int_{\mathbb{A}} \rho_{\mathcal{G}_{\ell}}^{m}(\mathbf{x}) m^{m}(d \mathbf{x})=\sum_{\mathbf{i} \in \mathbb{I}_{\ell}(\mathbb{A})} \rho_{\mathbb{F}(\ell)}^{m}(\mathbf{i}) .
$$

Let $\mathrm{I}(\ell)$ be the single configuration space over $\mathbb{I}(\ell)$. We write $i \in \mathrm{i}$ if $\mathrm{i}(\{i\})=1$. Each $\mathrm{i}=\sum_{i \in \mathrm{i}} \delta_{i} \in \mathrm{I}(\ell)$ can be regarded as a subset of $\mathbb{I}(\ell)$ by the correspondence of $\mathrm{i}$ to $\{i\}_{i \in \mathrm{i}}$. Let

$$
\Omega(\ell):=\bigcup_{i \in \mathbb{I}(\ell)}\{i\} \times \mathcal{B}_{\ell, i} .
$$


Let $\underline{\Omega}(\ell)$ be the single configuration space over $\Omega(\ell)$. Then by definition each element $\omega \in \underline{\Omega}(\ell)$ is of the form $\omega=\sum_{i \in \mathrm{i}} \delta_{\left(i, s_{i}\right)}$ such that $s_{i} \in \mathcal{B}_{\ell, i}$. Hence

$$
\underline{\Omega}(\ell) \subset\left\{\omega=\sum_{i \in \mathrm{i}} \delta_{\left(i, s_{i}\right)} ; \mathrm{i}=\sum_{i \in \mathrm{i}} \delta_{i} \in \mathrm{I}(\ell), s_{i} \in \mathcal{B}_{\ell, i}\right\} .
$$

Let $\mathrm{m}_{f_{\ell, i}}$ be as in (2.22). We set

$$
\mathrm{m}_{\mathbb{F}(\ell)}=\prod_{i \in \mathbb{I}(\ell)} \mathrm{m}_{f_{\ell, i}}, \quad \mathrm{~m}_{f_{\ell, \mathrm{i}}}=\prod_{i \in \mathrm{i}} \mathrm{m}_{f_{\ell, i}} .
$$

Remark 2 Let $\mathbf{i}=\left(i_{1}, \ldots, i_{m}\right)$ and $\mathbf{i}=\sum_{n=1}^{m} \delta_{i_{n}} \equiv \sum_{i \in \mathrm{i}} \delta_{i}$. By definition $\mathrm{m}_{f_{\ell, \mathrm{i}}}$ in (2.29) is a product measure on the product space $\prod_{i \in \mathrm{i}} \mathcal{B}_{\ell, i}$ with (unordered) parameter $i \in \mathrm{i}$, whereas $\mathrm{m}_{f_{\ell, \mathbf{i}}}$ in (2.23) is a product measure on the product space $\mathcal{B}_{i_{1}} \times \cdots \times \mathcal{B}_{i_{m}}$ with (ordered) parameter $\mathbf{i}=\left(i_{1}, \ldots, i_{m}\right)$.

We set $\iota_{\ell}: \underline{\Omega}(\ell) \rightarrow \mathrm{I}(\ell)$ such that $\iota_{\ell}(\omega)=\mathrm{i}$, and $\kappa_{\ell, \mathrm{i}}: \underline{\Omega}(\ell) \rightarrow \prod_{i \in \mathrm{i}} \mathcal{B}_{\ell, i}$ such that $\kappa_{\ell, \mathrm{i}}(\omega)=\left(s_{i}\right)_{i \in \mathrm{i}}$, where $\omega=\sum_{i \in \mathrm{i}} \delta_{\left(i, s_{i}\right)}, \mathrm{i}=\sum_{i \in \mathrm{i}} \delta_{i}$, and $s_{i} \in \mathcal{B}_{\ell, i}$.

Let $v_{\mathbb{F}(\ell)} \diamond \mathrm{m}_{\mathbb{F}(\ell)}$ be the probability measure on $\underline{\Omega}(\ell)$ given by

$$
\begin{aligned}
& \left(v_{\mathbb{F}(\ell)} \diamond \mathrm{m}_{\mathbb{F}(\ell)}\right) \circ \iota_{\ell}^{-1}(d \mathrm{i})=v_{\mathbb{F}(\ell)}(d \mathrm{i}), \\
& v_{\mathbb{F}(\ell)} \diamond \mathrm{m}_{\mathbb{F}(\ell)}\left(\kappa_{\ell, \mathrm{i}}(\omega) \in d \mathbf{s} \mid \iota_{\ell}(\omega)=\mathrm{i}\right)=\mathrm{m}_{f_{\ell, \mathrm{i}}}(d \mathbf{s}), \quad \mathbf{s}=\left(s_{i}\right)_{i \in \mathrm{i}} .
\end{aligned}
$$

Remark 3 (1) We can naturally regard the probability measures in (2.31) as a point process on $\prod_{i \in \mathrm{i}} \mathcal{B}_{\ell, i}$ supported on the set of configurations with exactly one particle configuration $\mathrm{S}=\delta_{\mathbf{s}}$ on $\prod_{i \in \mathrm{i}} \mathcal{B}_{\ell, i}$, that is, $\mathbf{s}=\left(s_{i}\right)_{i \in \mathrm{i}}$ is such that $s_{i} \in \mathcal{B}_{\ell, i}$ for each $i \in \mathrm{i}$.

(2) We can regard $\nu_{\mathbb{F}(\ell)} \diamond \mathrm{m}_{\mathbb{F}(\ell)}$ as a marked point process as follows: The configuration $i$ is distributed according to $v_{\mathbb{F}(\ell)}$, while the marks are independent and for each $\mathrm{i}$ the mark $\mathbf{s}$ is distributed according to $\mathrm{m}_{f_{\ell, \mathrm{i}}}$. Thus the space of marks depends on $\mathrm{i}$.

Theorem 3 Let $\mathfrak{u}_{\ell}: \underline{\Omega}(\ell) \rightarrow \mathrm{S}$ be such that $\mathfrak{u}_{\ell}(\omega)=\sum_{i \in \mathrm{i}} \delta_{s_{i}}$, where $\omega=\sum_{i \in \mathrm{i}} \delta_{\left(i, s_{i}\right)}$. Then

$$
\left.\mu\right|_{\mathcal{G}_{\ell}}=\left.\left(v_{\mathbb{F}(\ell)} \diamond \mathrm{m}_{\mathbb{F}(\ell)}\right) \circ \mathfrak{u}_{\ell}^{-1}\right|_{\mathcal{G}_{\ell}} .
$$

Remark 4 Theorem 3 implies that $\nu_{\mathbb{F}(\ell)} \diamond \mathrm{m}_{\mathbb{F}(\ell)}$ is a lift of $\left.\mu\right|_{\mathcal{G}_{\ell}}$ onto $\underline{\Omega}(\ell)$. We can naturally regard $\widetilde{\mathbb{I}}(\ell)$ as binary trees. Hence we call $\nu_{\mathbb{F}(\ell)} \diamond \mathrm{m}_{\mathbb{F}(\ell)}$ a tree representation of $\mu$ of level $\ell$.

We present a decomposition of $\left.\mu\right|_{\mathcal{G}_{\ell}}$, which follows from Theorem 3 immediately. Let $\mathrm{m}_{f_{\ell, \mathrm{i}}}^{\mathfrak{u}}=\mathrm{m}_{f_{\ell, \mathrm{i}}} \circ \mathfrak{u}_{\ell, \mathrm{i}}^{-1}$, where $\mathfrak{u}_{\ell, \mathrm{i}}: \prod_{i \in \mathrm{i}} \mathcal{B}_{\ell, i} \rightarrow \mathrm{S}$ is the unlabel map such that $\mathfrak{u}_{\ell, \mathrm{i}}\left(\left(s_{i}\right)_{i \in \mathrm{i}}\right)=$ $\sum_{i \in \mathrm{i}} \delta_{s_{i}}$.

Theorem 4 For each $\mathrm{A} \in \mathcal{G}_{\ell}$,

$$
\mu(\mathrm{A})=\int_{\mathrm{I}(\ell)} v_{\mathbb{F}(\ell)}(d \mathrm{i}) \mathrm{m}_{f_{\ell, \mathrm{i}}}^{\mathfrak{u}}(\mathrm{A}) .
$$

Let $\mathbb{I}(\ell)_{p}=\left\{i \in \mathbb{I}(\ell) ; r \leq p,\left|j_{1}\right| \leq p\right\}$, where $i=\left(\mathbf{j}_{\ell}, j_{\ell+1}, \ldots, j_{\ell+r-1}\right), r=\operatorname{rank}(i)$, and $\mathbf{j} \ell=\left(j_{1}, j_{2}, \ldots, j_{\ell}\right)$. Let $\pi_{p}^{c}(\mathrm{i})=\mathrm{i}\left(\cdot \cap \mathbb{I}(\ell)_{p}^{c}\right)$. Then we set Tail $(\mathbb{I}(\ell))=\cap_{p=1}^{\infty} \sigma\left[\pi_{p}^{c}\right]$. From this we can define the tail $\sigma$-field Tail $(\underline{\Omega}(\ell))$ of $\underline{\Omega}(\ell)$ because $\Omega(\ell)$ is a subset of $\mathbb{I}(\ell) \times S$.

Theorem $5 v_{\mathbb{F}(\ell)} \diamond \mathrm{m}_{\mathbb{F}(\ell)}$ is trivial on $\operatorname{Tail}(\underline{\Omega}(\ell)) \cap \mathfrak{u}_{\ell}^{-1}\left(\mathcal{G}_{\ell}\right)$. That is,

$$
\nu_{\mathbb{F}(\ell)} \diamond \mathrm{m}_{\mathbb{F}(\ell)}(\mathrm{A}) \in\{0,1\} \quad \text { for all } \mathrm{A} \in \operatorname{Tail}(\underline{\Omega}(\ell)) \cap \mathfrak{u}_{\ell}^{-1}\left(\mathcal{G}_{\ell}\right) .
$$


We remark that $\left.\mu\right|_{\mathcal{G}_{\ell}}$ is not a determinantal point process. Hence we exploit $v_{\mathbb{F}(\ell)} \diamond \mathrm{m}_{\mathbb{F}(\ell)}$ instead of $\left.\mu\right|_{\mathcal{G}_{\ell}}$. As we have seen in Theorem 3, $v_{\mathbb{F}(\ell)} \diamond \mathrm{m}_{\mathbb{F}(\ell)}$ is a lift of $\left.\mu\right|_{\mathcal{G}_{\ell}}$ in the sense of (2.32), from which we can deduce nice properties of $\left.\mu\right|_{\mathcal{G}_{\ell}}$. Indeed, an application of Theorem 3 combined with Theorem 5 is tail triviality of $\left.\mu\right|_{\mathcal{G}_{\ell}}$ :

Theorem $\left.6 \mu\right|_{\mathcal{G}_{\ell}}$ is tail trivial. That is,

$$
\left.\mu\right|_{\mathcal{G}_{\ell}}(\mathrm{B}) \in\{0,1\} \quad \text { for all } \mathrm{B} \in \operatorname{Tail}(\mathrm{S}) \cap \mathcal{G}_{\ell} .
$$

We shall apply Theorem 6 to prove Theorem 1 in Sect. 5 .

\section{Proof of Theorem 2}

The purpose of this section is to prove Theorem 2. In Lemma 1, we present the identity of kernels $\mathrm{K}$ and $\mathrm{K}_{\mathbb{F}(\ell)}$ using the orthonormal basis $\mathbb{F}(\ell)$, where $\mathrm{K}_{\mathbb{F}(\ell)}$ is the kernel given by (2.21) and $\mathbb{F}(\ell)$ is as in (2.15) and (2.16). In Lemma 2, we prove $\left(\mathrm{K}_{\mathbb{F}(\ell)}, \lambda_{\mathbb{I}(\ell)}\right)$ is a determinantal kernel and the associated determinantal point process $\nu_{\mathbb{F}(\ell)}$ exists. We will lift the the identity between $\mathrm{K}$ and $\mathrm{K}_{\mathbb{F}(\ell)}$ to that of correlation functions of $\left.\mu\right|_{\mathcal{G}_{\ell}}$ and $\nu_{\mathbb{F}(\ell)}$ in Theorem 2.

By definition $\mathbb{F}(\ell)=\left\{f_{\ell, i}\right\}_{i \in \mathbb{I}(\ell)}$ satisfies

$$
\begin{aligned}
& \int_{S}\left|f_{\ell, i}(x)\right|^{2} \mathrm{~m}(d x)=1 \quad \text { for all } i \in \mathbb{I}(\ell), \\
& \int_{S} f_{\ell, i}(x) \overline{f_{\ell, j}(x)} \mathrm{m}(d x)=0 \quad \text { for all } i \neq j \in \mathbb{I}(\ell) .
\end{aligned}
$$

Lemma 1 (1) Let $P(x)=\sum_{i} \xi(i) f_{\ell, i}(x)$ and $Q(y)=\sum_{j} \eta(j) f_{\ell, j}(y)$. Suppose that the supports of $\xi$ and $\eta$ are finite sets. Then

$$
\int_{S \times S} \mathrm{~K}(x, y) \overline{P(x)} Q(y) \mathrm{m}(d x) \mathrm{m}(d y)=\sum_{i, j} \mathrm{~K}_{\mathbb{F}(\ell)}(i, j) \overline{\xi(i)} \eta(j) .
$$

(2) We have an expansion of $\mathrm{K}$ in $L_{\mathrm{loc}}^{2}(S \times S, \mathrm{~m} \times \mathrm{m})$ such that

$$
\mathrm{K}(x, y)=\sum_{i, j \in \mathbb{I}(\ell)} \mathrm{K}_{\mathbb{F}(\ell)}(i, j) f_{\ell, i}(x) \overline{f_{\ell, j}(y)}
$$

Proof From (2.21) we deduce that

$$
\begin{aligned}
& \int_{S \times S} \mathrm{~K}(x, y) \overline{P(x)} Q(y) \mathrm{m}(d x) \mathrm{m}(d y) \\
& =\int_{S \times S} \mathrm{~K}(x, y) \overline{\sum_{i} \xi(i) f_{\ell, i}(x)} \sum_{j} \eta(j) f_{\ell, j}(x) \mathrm{m}(d x) \mathrm{m}(d y) \\
& =\sum_{i, j} \int_{S \times S} \mathrm{~K}(x, y) \overline{f_{\ell, i}(x)} f_{\ell, j}(y) \mathrm{m}(d x) \mathrm{m}(d y) \overline{\xi(i)} \eta(j) \\
& =\sum_{i, j} \mathrm{~K}_{\mathbb{F}(\ell)}(i, j) \overline{\xi(i)} \eta(j)
\end{aligned}
$$


This yields (3.3). We have thus proved (1). By a direct calculation, we have

$$
\begin{aligned}
& \int_{S} P(x) \overline{f_{\ell, i}(x)} \mathrm{m}(d x)=\int_{S} \sum_{p} \xi(p) f_{\ell, p}(x) \overline{f_{\ell, i}(x)} \mathrm{m}(d x)=\xi(i), \\
& \int_{S} Q(y) \overline{f_{\ell, j}(y)} \mathrm{m}(d y)=\int_{S} \sum_{q} \eta(q) f_{\ell, q}(y) \overline{f_{\ell, j}(y)} \mathrm{m}(d y)=\eta(j) .
\end{aligned}
$$

Combining (3.5) and (3.6) yields

$$
\begin{aligned}
& \int_{S \times S} \mathrm{~K}(x, y) \overline{P(x)} Q(y) \mathrm{m}(d x) \mathrm{m}(d y)= \\
& \int_{S \times S} \sum_{i, j} \mathrm{~K}_{\mathbb{F}(\ell)}(i, j) f_{\ell, i}(x) \overline{f_{\ell, j}(y) P(x)} Q(y) \mathrm{m}(d x) \mathrm{m}(d y) .
\end{aligned}
$$

This implies (3.4).

Let $\lambda_{\mathbb{I}(\ell)}$ be the counting measure on $\mathbb{I}(\ell)$ as before. We can regard $\mathrm{K}_{\mathbb{F}(\ell)}$ as an operator on $L^{2}\left(\mathbb{I}(\ell), \lambda_{\mathbb{I}(\ell)}\right)$ such that $\mathrm{K}_{\mathbb{F}(\ell)} \xi(i)=\sum_{j \in \mathbb{I}(\ell)} \mathrm{K}_{\mathbb{F}(\ell)}(i, j) \xi(j)$. We now prove that the

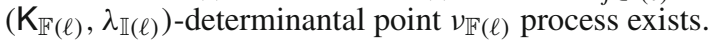

Lemma 2 Let $\operatorname{Spec}\left(\mathrm{K}_{\mathbb{F}(\ell)}\right)$ be the spectrum of $\mathrm{K}_{\mathbb{F}(\ell)}$. Then

$$
\operatorname{Spec}\left(\mathrm{K}_{\mathbb{F}(\ell)}\right) \subset[0,1] .
$$

In particular, there exists a unique, determinantal point process $v_{\mathbb{F}(\ell)}$ on $\mathbb{I}(\ell)$ associated with $\left(\mathrm{K}_{\mathbb{F}(\ell)}, \lambda_{\mathbb{I}(\ell)}\right)$.

Proof Recall that $\mathbb{F}(\ell)=\left\{f_{\ell, i}\right\}_{i \in \mathbb{I}(\ell)}$ is an orthonormal basis of $L^{2}(S, \mathrm{~m})$. Let $U$ : $L^{2}(S, \mathrm{~m}) \rightarrow L^{2}\left(\mathbb{I}(\ell), \lambda_{\mathbb{I}(\ell)}\right)$ be the unitary operator such that $U\left(f_{\ell, i}\right)=e_{\ell, i}$, where $\left\{e_{\ell, i}\right\}_{i \in \mathbb{I}(\ell)}$ is the canonical orthonormal basis of $L^{2}(\mathbb{I}(\ell), \lambda \mathbb{I}(\ell)$. Then by Lemma 1 we see that $\mathrm{K}_{\mathbb{F}(\ell)}=U \mathrm{KU}^{-1}$. Hence $\mathrm{K}_{\mathbb{F}(\ell)}$ and $\mathrm{K}$ have the same spectrum. We thus obtain (3.7). Because $\mathrm{K}_{\mathbb{F}(\ell)}$ is Hermitian symmetric, the second claim is clear from (3.7), (A1), and general theory [10-12].

Lemma 3 Let $\mathcal{B}_{\ell, i}=\operatorname{supp}\left(f_{\ell, i}\right)$ be as in (2.19). Then, for $i, j \in \mathbb{I}(\ell)$ and $\mathcal{A} \in \mathcal{F}_{\ell}$,

$$
\int_{\mathcal{A}} f_{\ell, i}(x) \overline{f_{\ell, j}(x)} \mathrm{m}(d x)=\left\{\begin{array}{ll}
1 & \left(i=j, \mathcal{B}_{\ell, i} \subset \mathcal{A}\right) \\
0 & \text { (otherwise })
\end{array} .\right.
$$

Proof We recall that $\mathcal{B}_{\ell, i}$ is the support of $f_{\ell, i}$ by (2.19). Suppose $i=j$ and $\mathcal{B}_{\ell, i} \subset \mathcal{A}$. Then from (3.1)

$$
\int_{\mathcal{A}} f_{\ell, i}(x) \overline{f_{\ell, j}(x)} \mathrm{m}(d x)=\int_{S} f_{\ell, i}(x) \overline{f_{\ell, i}(x)} \mathrm{m}(d x)=1 .
$$

Suppose that $i=j$ and that $\mathcal{B}_{\ell, i} \not \subset \mathcal{A}$. Then, using $\mathcal{A} \in \mathcal{F}_{\ell},(2.7)$, and (2.20), we deduce that $\mathcal{B}_{\ell, i} \cap \mathcal{A}=\emptyset$. Because $\mathcal{B}_{\ell, i}=\operatorname{supp}\left(f_{\ell, i}\right)$, we obtain

$$
\int_{\mathcal{A}} f_{\ell, i}(x) \overline{f_{\ell, j}(x)} \mathrm{m}(d x)=0 .
$$

Finally, suppose $i \neq j$. Because $\mathcal{A} \in \mathcal{F}_{\ell}$, we see that $\mathcal{B}_{\ell, i} \subset \mathcal{A}$ or $\mathcal{B}_{\ell, i} \cap \mathcal{A}=\emptyset$. The same also holds for $\mathcal{B}_{\ell, j}$. In any case, we obtain (3.10) from (3.2). From (3.9) and (3.10), we obtain (3.8). 
Proof of Theorem 2 Let $\mathbb{A}=\mathcal{A}_{1} \times \cdots \times \mathcal{A}_{m} \in \mathcal{A}_{n}$ as in Theorem 2. Then, because $\mathcal{A}_{n} \in \Delta(\ell)$ for all $n=1, \ldots, m$, we deduce from (1.1) and (3.4) that

$$
\begin{aligned}
\int_{\mathbb{A}} \rho_{\mathcal{G}_{\ell}}^{m}(\mathbf{x}) \mathrm{m}^{m}(d \mathbf{x}) \\
=\int_{\mathbb{A}} \rho^{m}(\mathbf{x}) \mathrm{m}^{m}(d \mathbf{x}) \\
=\int_{\mathbb{A}} \operatorname{det}\left[\sum_{i, j \in \mathbb{I}(\ell)} \mathrm{K}_{\mathbb{F}(\ell)}(i, j) f_{\ell, i}\left(x_{p}\right) \overline{f_{\ell, j}\left(x_{q}\right)}\right]_{p, q=1}^{m} \mathrm{~m}^{m}(d \mathbf{x}),
\end{aligned}
$$

where $\mathbf{x}=\left(x_{1}, \ldots, x_{m}\right)$. From a straightforward calculation and Lemma 1, we obtain

$$
\begin{aligned}
& \int_{\mathbb{A}} \operatorname{det}\left[\sum_{i, j \in \mathbb{I}(\ell)} \mathrm{K}_{\mathbb{F}(\ell)}(i, j) f_{\ell, i}\left(x_{p}\right) \overline{f_{\ell, j}\left(x_{q}\right)}\right]_{p, q=1}^{m} \mathrm{~m}^{m}(d \mathbf{x}) \\
& =\int_{\mathbb{A}} \sum_{\sigma \in \mathfrak{S}_{m}} \operatorname{sgn}(\sigma) \prod_{p=1}^{m}\left(\sum_{i_{p}, j_{p} \in \mathbb{I}(\ell)} \mathrm{K}_{\mathbb{F}(\ell)}\left(i_{p}, j_{p}\right) f_{\ell, i_{p}}\left(x_{p}\right) \overline{f_{\ell, j_{p}}\left(x_{\sigma(p)}\right)}\right) \mathrm{m}^{m}(d \mathbf{x}) \\
& =\sum_{\sigma \in \mathfrak{S}_{m}} \operatorname{sgn}(\sigma) \int_{\mathbb{A}} \prod_{p=1}^{m}\left(\sum_{i_{p}, j_{p} \in \mathbb{I}(\ell)} \mathrm{K}_{\mathbb{F}(\ell)}\left(i_{p}, j_{p}\right) f_{\ell, i_{p}}\left(x_{p}\right) \overline{f_{\ell, j_{p}}\left(x_{\sigma(p)}\right)}\right) \mathrm{m}^{m}(d \mathbf{x}) \\
& =\sum_{\sigma \in \mathfrak{S}_{m}} \operatorname{sgn}(\sigma) \lim _{R \rightarrow \infty} \int_{\mathbb{A}} \prod_{p=1}^{m}\left(\sum_{i_{p}, j_{p} \in \mathbb{I}(\ell ; R)} \mathrm{K}_{\mathbb{F}(\ell)}\left(i_{p}, j_{p}\right) f_{\ell, i_{p}}\left(x_{p}\right) \overline{f_{\ell, j_{p}}\left(x_{\sigma(p)}\right)}\right) \mathrm{m}^{m}(d \mathbf{x}) \\
& =\sum_{\sigma \in \mathfrak{S}_{m}} \operatorname{sgn}(\sigma) \lim _{R \rightarrow \infty} \int_{\mathbb{A}}\left(\sum_{i, \mathbf{j} \in \mathbb{I}(\ell ; R)^{m}} \prod_{p=1}^{m} \mathrm{~K}_{\mathbb{F}(\ell)}\left(i_{p}, j_{p}\right) f_{\ell, i_{p}}\left(x_{p}\right) \overline{f_{\ell, j_{p}}\left(x_{\sigma(p)}\right)}\right) \mathrm{m}^{m}(d \mathbf{x}),
\end{aligned}
$$

where $\mathbb{I}(\ell ; R)=\{i \in \mathbb{I}(\ell) ; \operatorname{rank}(i) \leq R\}$ and $\operatorname{rank}(i)$ is defined before (2.15). Furthermore, $\mathbf{i}=\left(i_{1}, \ldots, i_{m}\right), \mathbf{j}=\left(j_{1}, \ldots, j_{m}\right) \in \mathbb{I}(\ell)^{m}$. We note that $\cup_{i=1}^{m} \mathcal{A}_{i}$ is relatively compact. Hence the fourth line in (3.12) follows from Lemma 1(2) and the Schwarz inequality. Using Lemma 3 we obtain

$$
\begin{aligned}
& \int_{\mathbb{A}}\left(\sum_{\mathbf{i}, \mathbf{j} \in \mathbb{I}(\ell ; R)^{m}} \prod_{p=1}^{m} \mathrm{~K}_{\mathbb{F}(\ell)}\left(i_{p}, j_{p}\right) f_{\ell, i_{p}}\left(x_{p}\right) \overline{f_{\ell, j_{p}}\left(x_{\sigma(p)}\right)}\right) \mathrm{m}^{m}(d \mathbf{x})
\end{aligned}
$$

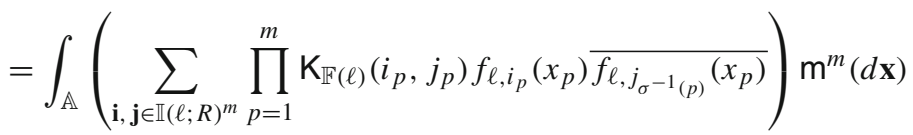

$$
\begin{aligned}
& =\int_{\mathbb{A}}\left(\sum_{\mathbf{i} \in \mathbb{I}(\ell ; R)^{m}} \prod_{p=1}^{m} \mathrm{~K}_{\mathbb{F}(\ell)}\left(i_{p}, i_{\sigma(p)}\right)\left|f_{\ell, i_{p}}\left(x_{p}\right)\right|^{2}\right) \mathrm{m}^{m}(d \mathbf{x}) \\
& \rightarrow \int_{\mathbb{A}}\left(\sum_{\mathbf{i} \in \mathbb{I}(\ell)^{m}} \prod_{p=1}^{m} \mathrm{~K}_{\mathbb{F}(\ell)}\left(i_{p}, i_{\sigma(p)}\right)\left|f_{\ell, i_{p}}\left(x_{p}\right)\right|^{2}\right) \mathrm{m}^{m}(d \mathbf{x}) \quad \text { as } R \rightarrow \infty .
\end{aligned}
$$


The convergence in the last line follows from Lemma 1(2) and the Schwarz inequality again. Multiplying $\operatorname{sgn}(\sigma)$ and taking summation over $\sigma \in \mathfrak{S}_{m}$ in the last line, we deduce from (2.22)-(2.24) that

$$
\begin{aligned}
& \sum_{\sigma \in \mathbb{S}_{m}} \operatorname{sgn}(\sigma) \int_{\mathbb{A}}\left(\sum_{\mathbf{i} \in \mathbb{I}(\ell)^{m}} \prod_{p=1}^{m} \mathrm{~K}_{\mathbb{F}(\ell)}\left(i_{p}, i_{\sigma(p)}\right)\left|f_{\ell, i_{p}}\left(x_{p}\right)\right|^{2}\right) \mathrm{m}^{m}(d \mathbf{x}) \\
& =\int_{\mathbb{A}} \sum_{\mathbf{i} \in \mathbb{I}(\ell)^{m}} \operatorname{det}\left[\mathrm{K}_{\mathbb{F}(\ell)}\left(i_{p}, i_{q}\right)\right]_{p, q=1}^{m}\left\{\prod_{p=1}^{m}\left|f_{\ell, i_{p}}\left(x_{p}\right)\right|^{2}\right\} \mathrm{m}^{m}(d \mathbf{x}) \\
& =\int_{\mathbb{A}} \sum_{\mathbf{i} \in \mathbb{I}(\ell)^{m}} \rho_{\mathbb{F}(\ell)}^{m}(\mathbf{i}) \mathrm{m}_{f_{\ell, \mathbf{i}}}(d \mathbf{x}) \\
& =\sum_{\mathbf{i} \in \in \mathbb{I}_{\ell}(\mathbb{A})} \rho_{\mathbb{F}(\ell)}^{m}(\mathbf{i}) .
\end{aligned}
$$

Combining (3.11)-(3.14) we deduce (2.26), which completes the proof.

\section{Proof of Theorems 3-6}

In this section, we prove Theorem 3-Theorem 6.

\subsection{Proof of Theorem 3}

Let $\varrho^{m}$ be the $m$-point correlation function of $\left.\left(\nu_{\mathbb{F}(\ell)} \diamond \mathrm{m}_{f_{\ell}}\right) \circ \mathfrak{u}_{\ell}^{-1}\right|_{\mathcal{G}_{\ell}}$. Then it suffices for (2.32) to prove

$$
\rho_{\mathcal{G}_{\ell}}^{m}(\mathbf{x})=\varrho^{m}(\mathbf{x}) .
$$

From (1.5) and $\mathcal{F}_{\ell}=\sigma\left[\mathcal{A}_{\ell, i} ; i \in I(\ell)\right]$, we see that $\rho_{\mathcal{G}_{\ell}}^{m}$ and $\varrho^{m}$ are $\mathcal{F}_{\ell}^{m}$-measurable. Let $m=m_{1}+\cdots+m_{k}$. Let $\mathbb{A}=\mathcal{A}_{1}^{m_{1}} \times \cdots \times \mathcal{A}_{k}^{m_{k}} \in \Delta(\ell)^{m}$ such that $\mathcal{A}_{p} \cap \mathcal{A}_{q}=\emptyset$ if $p \neq q$. Let $\mathbf{i}=\left(i_{n}\right)_{n=1}^{m}=\left(\mathbf{i}_{1}, \ldots, \mathbf{i}_{k}\right) \in \mathbb{I}(\ell)^{m}$ such that $\mathbf{i}_{n} \in \mathbb{I}(\ell)^{m_{n}}$. From Theorem 2 , we see that

$$
\int_{\mathbb{A}} \rho_{\mathcal{G}_{\ell}}^{m}(\mathbf{x}) \mathrm{m}^{m}(d \mathbf{x})=\sum_{\mathbf{i} \in \mathbb{I}_{\ell}(\mathbb{A})} \rho_{\mathbb{F}(\ell)}^{m}(\mathbf{i}) .
$$

By the definition of correlation functions, (2.30), and (2.31), we see that

$$
\begin{aligned}
\sum_{\mathbf{i} \in \mathbb{I}_{\ell}(\mathbb{A})} \rho_{\mathbb{F}(\ell)}^{m}(\mathbf{i}) & =\int_{\mathbb{I}(\ell)} \prod_{n=1}^{k} \frac{\mathrm{i}\left(\mathbb{I}_{\ell}\left(\mathcal{A}_{n}\right)\right) !}{\left(\mathbf{i}\left(\mathbb{I}_{\ell}\left(\mathcal{A}_{n}\right)\right)-m_{n}\right) !} v_{\mathbb{F}(\ell)}(d \mathbf{i}) \\
& =\left.\int_{\mathbf{S}} \prod_{n=1}^{k} \frac{\mathrm{s}\left(\mathcal{A}_{n}\right) !}{\left(\mathbf{s}\left(\mathcal{A}_{n}\right)-m_{n}\right) !}\left(\nu_{\mathbb{F}(\ell)} \diamond \mathrm{m}_{f_{\ell}}\right) \circ \mathfrak{u}_{\ell}^{-1}\right|_{\mathcal{G}_{\ell}}(d \mathbf{s}) \\
& =\int_{\mathbb{A}} \varrho^{m}(\mathbf{x}) \mathrm{m}^{m}(d \mathbf{x}) .
\end{aligned}
$$

Combining (4.2) and (4.3), we deduce that

$$
\int_{\mathbb{A}} \rho_{\mathcal{G}_{\ell}}^{m}(\mathbf{x}) \mathrm{m}^{m}(d \mathbf{x})=\sum_{\mathbf{i} \in \mathbb{I}_{\ell}(\mathbb{A})} \rho_{\mathbb{F}(\ell)}^{m}(\mathbf{i})=\int_{\mathbb{A}} \varrho^{m}(\mathbf{x}) \mathrm{m}^{m}(d \mathbf{x}) .
$$


From (4.4), we obtain (4.1). This completes the proof of Theorem 3.

\subsection{Proof of Theorem 4}

Theorem 4 follows from Theorem 3 immediately.

\subsection{Proof of Theorem 5}

It is known that determinantal point processes on discrete spaces are tail trivial $[7,11]$. Hence $\nu_{\mathbb{F}(\ell)}$ is tail trivial by Lemma 2 .

Let $\mathfrak{u}_{\ell}$ be as in Theorem 3. Let $A \in \mathfrak{u}_{\ell}^{-1}\left(\mathcal{G}_{\ell}\right)$. Then there exists a $\mathrm{B} \in \mathcal{B}(\mathrm{I}(\ell))$ such that $A=\iota_{\ell}^{-1}(B)$. From this we deduce that, for each $A \in \operatorname{Tail}(\underline{\Omega}(\ell)) \cap \mathfrak{u}_{\ell}^{-1}\left(\mathcal{G}_{\ell}\right)$, there exists a $\mathrm{B} \in \operatorname{Tail}(\mathrm{I}(\ell))$ such that $\mathrm{A}=\iota_{\ell}^{-1}(\mathrm{~B})$. Hence from (2.30) we deduce

$$
v_{\mathbb{F}(\ell)} \diamond \mathrm{m}_{\mathbb{F}(\ell)}(\mathrm{A})=v_{\mathbb{F}(\ell)}(\mathrm{B}) .
$$

From (4.5) and tail triviality of $v_{\mathbb{F}(\ell)}$ we deduce that

$$
\nu_{\mathbb{F}(\ell)} \diamond \mathrm{m}_{\mathbb{F}(\ell)}(\mathrm{A}) \in\{0,1\}
$$

for each $\mathrm{A} \in \operatorname{Tail}(\underline{\Omega}(\ell)) \cap \mathfrak{u}_{\ell}^{-1}\left(\mathcal{G}_{\ell}\right)$. We easily see that $\mathfrak{u}_{\ell}^{-1}\left(\mathcal{G}_{\ell}\right) \subset \sigma\left[\iota_{\ell}\right]$. Hence

$$
\operatorname{Tail}(\underline{\Omega}(\ell)) \cap \mathfrak{u}_{\ell}^{-1}\left(\mathcal{G}_{\ell}\right) \subset \operatorname{Tail}(\underline{\Omega}(\ell)) \cap \sigma[\iota \ell] .
$$

Combining (4.6) and (4.7) completes the proof of Theorem 5.

\subsection{Proof of Theorem 6}

Let $\mathrm{B} \in \operatorname{Tail}(\mathrm{S}) \cap \mathcal{G}_{\ell}$. Then we deduce that

$$
\mathfrak{u}_{\ell}^{-1}(\mathrm{~B}) \in \operatorname{Tail}(\underline{\Omega}(\ell)) \cap \mathfrak{u}_{\ell}^{-1}\left(\mathcal{G}_{\ell}\right) .
$$

Hence from Theorems 3 and 5, we deduce that

$$
\mu(\mathrm{B})=\left.\mu\right|_{\mathcal{G}_{\ell}}(\mathrm{B})=v_{\mathbb{F}(\ell)} \diamond \mathrm{m}_{\mathbb{F}(\ell)}\left(\mathfrak{u}_{\ell}^{-1}(\mathrm{~B})\right) \in\{0,1\} .
$$

This completes the proof.

\section{Proof of Theorem 1}

In this section, we complete the proof of Theorem 1.

Lemma 4 Let $X$ be a Tail(S)-measurable and integrable random variable. Then $E^{\mu}\left[X \mid \mathcal{G}_{\ell}\right]$ is Tail(S) $\cap \mathcal{G}_{\ell}$-measurable.

Proof Recall that $\Delta(\ell)=\left\{\mathcal{A}_{\ell, i}\right\}_{i \in I(\ell)}$. Let $\pi_{T_{r}}$ be the projection with $T_{r}$ such that

$$
T_{r}=\bigcup_{\substack{\mathcal{A}_{\ell, i} \cap S_{r} \neq \emptyset ; \\ i \in I(\ell)}} \mathcal{A}_{\ell, i} .
$$

Then $X \in L^{1}(\mathrm{~S}, \mu)$ is $\sigma\left[\pi_{T_{r}^{c}}\right]$-measurable because $X \in L^{1}(\mathrm{~S}, \mu)$ is Tail(S)-measurable and each $\mathcal{A}_{\ell, i}$ is relatively compact. Hence for each $r \in \mathbb{N}$

$$
X(\mathrm{~s})=X \circ \pi_{T_{r}^{c}}(\mathrm{~s}) .
$$


From this we deduce that

$$
E^{\mu}\left[X \mid \mathcal{G}_{\ell}\right]=E^{\mu}\left[X \circ \pi_{T_{r}^{c}} \mid \mathcal{G}_{\ell}\right] .
$$

By construction $S_{r} \subset T_{r}$. Then from this and (5.3) we see that $E^{\mu}\left[X \mid \mathcal{G}_{\ell}\right]$ is $\sigma\left[\pi_{S_{r}^{c}}\right]$ measurable for each $r \in \mathbb{N}$. Hence $E^{\mu}\left[X \mid \mathcal{G}_{\ell}\right]$ is Tail(S)-measurable because $\cap_{r \in \mathbb{N}} \sigma\left[\pi_{S_{r}^{c}}\right]=$ Tail(S). By construction $E^{\mu}\left[X \mid \mathcal{G}_{\ell}\right]$ is $\cap_{r \in \mathbb{N}} \sigma\left[\pi_{S_{r}^{c}}\right]$-measurable. Combining these completes the proof of Lemma 4

Lemma 5 For all $\mathrm{A} \in$ Tail(S)

$$
\mu(\mathrm{A})=\mu\left(\mathrm{A} \mid \mathcal{G}_{\ell}\right)(\mathrm{s}) \text { for } \mu \text {-a.s.s. }
$$

Proof From the definition of the conditional probability, we see that

$$
\mu(\mathrm{A})=\int_{\mathrm{S}} \mu\left(\mathrm{A} \mid \mathcal{G}_{\ell}\right)(\mathrm{s}) \mu(d \mathbf{s}) .
$$

From Lemma 4, we deduce that $\mu\left(\mathrm{A} \mid \mathcal{G}_{\ell}\right)(\mathrm{s})=E^{\mu}\left[1_{\mathrm{A}} \mid \mathcal{G}_{\ell}\right](\mathrm{s})$ is Tail(S) $\cap \mathcal{G}_{\ell}$-measurable. Hence from Theorem 6 we obtain that $\mu\left(\mathrm{A} \mid \mathcal{G}_{\ell}\right)(\mathrm{s})$ is constant $\mu$-a.s. $\mathrm{S}$. This combined with (5.5) yields (5.4).

Lemma 6 For each $\mathrm{A} \in \mathcal{B}(\mathrm{S})$

$$
\lim _{\ell \rightarrow \infty} \mu\left(\mathrm{A} \mid \mathcal{G}_{\ell}\right)(\mathbf{s})=1_{\mathrm{A}}(\mathrm{s}) \text { for } \mu \text {-a.s. s. }
$$

Proof From (1.6), we apply the martingale convergence theorem to obtain the convergence such that, for all $A \in \mathcal{B}(S)$,

$$
\lim _{\ell \rightarrow \infty} \mu\left(\mathrm{A} \mid \mathcal{G}_{\ell}\right)(\mathrm{s})=\lim _{\ell \rightarrow \infty} E^{\mu}\left[1_{\mathrm{A}} \mid \mathcal{G}_{\ell}\right](\mathrm{s})=E^{\mu}\left[1_{\mathrm{A}} \mid \mathcal{B}(\mathrm{S})\right](\mathrm{s})=1_{\mathrm{A}}(\mathrm{s})
$$

for $\mu$-a.s. S. We have thus proved (5.6).

Proof of Theorem 1 From Lemmas 5 and 6 we deduce that

$$
\mu(\mathrm{A})=\mu\left(\mathrm{A} \mid \mathcal{G}_{\ell}\right)(\mathrm{s}) \rightarrow_{\ell \rightarrow \infty} 1_{\mathrm{A}}(\mathrm{s}) \text { for } \mu \text {-a.s. } \mathrm{s} .
$$

Hence we obtain $\mu(\mathrm{A}) \in\{0,1\}$.

\section{Examples Related to Random Matrices}

In this section, we give typical examples of determinantal point processes related to random matrix theory [3,9]. All examples below are tail trivial because of Theorem 1 .

All the kernels $\mathrm{K}(x, y)$ below are continuous. In Examples 3-5, we define the kernels only off diagonal. On diagonal, they are defined by continuity.

Example 3 (sine point process) Let $\mathrm{S}=\mathbb{R}$ and $\mathrm{m}(d x)=d x$. Let

$$
\mathrm{K}_{\sin }(x, y)=\frac{\sin (x-y)}{\pi(x-y)} \quad(x \neq y)
$$

be the sine kernel. The associated determinantal point process $\mu_{\text {sin }}$ is called the sine 2 point process. 
Example 4 (Airy point process) Let $\mathrm{S}=\mathbb{R}$ and $\mathrm{m}(d x)=d x$. Let

$$
\mathrm{K}_{\mathrm{Ai}}(x, y)=\frac{\operatorname{Ai}(x) \operatorname{Ai}^{\prime}(y)-\operatorname{Ai}^{\prime}(x) \operatorname{Ai}(y)}{x-y}(x \neq y)
$$

be the Airy kernel. Here Ai is the Airy function, and $\mathrm{Ai}^{\prime}$ is its derivative. The associated determinantal point process $\mu_{\mathrm{Ai}}$ is called the Airy point process $[3,9]$.

Example 5 (Bessel point process) Let $\mathrm{S}=[0, \infty)$ and $\mathrm{m}(d x)=d x$. Let $1 \leq \alpha<\infty$. Let $\mathrm{K}_{\mathrm{Be}, \alpha}$ be the Bessel kernel such that

$$
\mathrm{K}_{\mathrm{Be}, \alpha}(x, y)=\frac{J_{\alpha}(\sqrt{x}) \sqrt{y} J_{\alpha}^{\prime}(\sqrt{y})-\sqrt{x} J_{\alpha}^{\prime}(\sqrt{x}) \sqrt{y} J_{\alpha}(\sqrt{y})}{2(x-y)} \quad(x \neq y) .
$$

Let $\mu_{\mathrm{Be}, \alpha}$ be the associated determinantal point process. $\mu_{\mathrm{Be}, \alpha}$ is called the $\mathrm{Bessel}_{2, \alpha}$ point process.

Example 6 (Ginibre point process) Let $\mathrm{S}=\mathbb{R}^{2}$ and $\mathrm{m}(d x)=(1 / \pi) e^{-|x|^{2}} d x$. Let $\mathrm{K}_{\text {Gin }}$ : $\mathbb{R}^{2} \times \mathbb{R}^{2} \rightarrow \mathbb{C}$ be the exponential kernel such that

$$
\mathrm{K}_{\mathrm{Gin}}(x, y)=e^{x \bar{y}} .
$$

Here we identify $\mathbb{R}^{2}$ as $\mathbb{C}$ by the obvious correspondence $\mathbb{R}^{2} \ni x=\left(x_{1}, x_{2}\right) \mapsto x_{1}+$ $\sqrt{-1} x_{2} \in \mathbb{C}$, and $\bar{y}=y_{1}-\sqrt{-1} y_{2}$ is the complex conjugate in this identification. The associated determinantal point process $\mu_{\text {Gin }}$ is called the Ginibre point process.

Acknowledgements This work was supported by JSPS KAKENHI Grant Numbers JP16K13764, JP16H02149, JP16H06338, and also in part by a Grant-in-Aid for Scientific Research (KIBAN-A, No. 24244010).

Open Access This article is distributed under the terms of the Creative Commons Attribution 4.0 International License (http://creativecommons.org/licenses/by/4.0/), which permits unrestricted use, distribution, and reproduction in any medium, provided you give appropriate credit to the original author(s) and the source, provide a link to the Creative Commons license, and indicate if changes were made.

\section{References}

1. Anderson, G.W., Guionnet, A., Zeitouni, O.: An Introduction to Random Matrices. Cambridge University Press, Cambridge (2010)

2. Bufetov, A.I., Qiu, Y., Shamov A.: Kernels of conditional determinantal measures. Preprint. arXiv:1612.06751 [math.PR]

3. Forrester, P.J.: Log-Gases and Random Matrices London Mathematical Society Monographs. Princeton University Press, Princeton (2010)

4. Ghosh, S.: Determinantal processes and completeness of random exponentials: the critical case. Probab. Theory Relat. Fields 163, 1-23 (2014)

5. Hough, B., Krishnapur, M., Peres, Y., Virág, B.: Zeros of Gaussian Analytic Functions and Determinantal Point Processes. University Lecture Series, vol. 51. AMS, Providence (2009)

6. Lyons, R., Steif, J.: Stationary determinantal processes: phase mutiplicity, Bernoullicity, entropy, and domination. Duke Math. J 130(3), 515-575 (2003)

7. Lyons, R.: Determinantal probability measures. Publ. Math. Inst. Hautes Études Sci. 98, 167-212 (2003)

8. Lyons, R.: Determinantal probability: basic properties and conjectures (2014). arXiv:1406.2707v1

9. Mehta, M.L.: Random Matrices, 3rd edn. Elsevier, Amsterdam (2004)

10. Soshnikov, A.: Determinantal random point fields. Russ. Math. Surv. 55, 923-975 (2000)

11. Shirai, T., Takahashi, Y.: Random point fields associated with certain Fredholm determinants I: Fermion, Poisson and Boson processes. J. Funct. Anal. 205, 414-463 (2003)

12. Shirai, T., Takahashi, Y.: Random point fields associated with certain Fredholm determinants II: fermion shifts and their ergodic properties. Ann. Probab. 31, 1533-1564 (2003) 